\title{
Study of the Sensing Characteristics of Irradiated Fiber Bragg Gratings and Fabry-Perot Interferometers Under Gamma Radiation
}

\author{
Zhuoyue $\mathrm{LI}^{1}$, Zengling RAN ${ }^{2}$, Xianguo QING ${ }^{1}$, Zhengxi $\mathrm{HE}^{1}$, Yaqin XIAO ${ }^{3}$, \\ Tingting $\mathrm{YANG}^{2}$, Xiu $\mathrm{HE}^{2}$, Jialiang $\mathrm{ZHU}^{1}$, Peng $\mathrm{HE}^{1}$, and Sijie $\mathrm{XU}^{1}$
}

\author{
${ }^{1}$ Science and Technology on Reactor System Design Technology Laboratory, Nuclear Power Institute of China, Chengdu \\ 610213, China \\ ${ }^{2}$ Key Laboratory of Optical Fiber Sensing \& Communications (Ministry of Education), University of Electronic Science \\ and Technology of China, Chengdu 611731, China \\ ${ }^{3}$ China Aerodynamics Research and Development Center, Mianyang 621000, China \\ *Corresponding author: Zengling RAN_ E-mail: ranzl@126.com
}

\begin{abstract}
The sensing characteristics of irradiated fiber Bragg gratings (FBGs) and Fabry-Perot interferometers (FPIs) were investigated under a $2 \mathrm{MGy}$ dose of gamma radiation. The study found that the pressure sensitivity of FP sensors after irradiation was stable, while the temperature sensitivity of FBG sensors was unstable, and both wavelengths displayed a shift. These findings offer the possibility for the application of FP pressure sensors in the gamma radiation environments, and FBG sensors require further research to be suitable for application in the nuclear radiation environments.
\end{abstract}

Keywords: Fiber Bragg gratings; Fabry-Perot interferometers; gamma radiation

Citation: Zhuoyue LI, Zengling RAN, Xianguo QING, Zhengxi HE, Yaqin XIAO, Tingting YANG, et al., "Study of the Sensing Characteristics of Irradiated Fiber Bragg Gratings and Fabry-Perot Interferometers Under Gamma Radiation," Photonic Sensors, 2022, 12(1): 91-98.

\section{Introduction}

Optical fiber sensors have drawn much attention due to their advantages under exposure to nuclear radiation, such as compact size, high sensitivity, wavelength multiplexing, and anti-electromagnetic interference [1-3].

Since the 1990s, researchers have been exploring the use of fiber Bragg gratings (FBGs) in radiation environments [4-6]. Radiation induced Bragg wavelength shift (RI-BWS) and radiation induced attenuation (RIA) were investigated by Remy et al.
[7], Xu et al. [8], Gusarov et al. [9], Henschel et al. $[10,11]$, and Perry et al. [12]. RI-BWS ranged from $20 \mathrm{pm}$ to $700 \mathrm{pm}$ with radiation doses ranging from $100 \mathrm{kGy}$ to $3.8 \mathrm{GGy}$. It was found that the RI-BWS is related to fiber doping, the FBG fabrication process, and radiation temperature. To improve the radiation resistance of FBGs, researchers have found that FBGs written on pure-silica-core fiber, nitrogen-doped fiber, and phosphorus-doped fiber have better radiation resistance than those written on ordinary single-mode fibers (SMF) [8-10]. Ten times of the RI-BWS variation was also observed

Received: 22 December 2020 / Revised: 7 February 2021

(C) The Author(s) 2021. This article is published with open access at Springerlink.com

DOI: 10.1007/s13320-021-0630-9

Article type: Regular 
with variations in the FBG fabrication process [10]. Environmental temperature during irradiation also strongly influenced RI-BWS. It was found that RI-BWS at $-50{ }^{\circ} \mathrm{C}$ was about two times higher than that at room temperature [11]. There has been little investigation of the effect of radiation on sensor temperature sensitivity.

Fabry-Perot interferometers (FPIs) sensors have also been investigated in the nuclear radiation environments. Liu et al. [13] found that gamma radiation had negligible effects on a capillary-based FP temperature sensor manufactured by Fiso technologies. The static calibration curves of the FP temperature sensor had excellent linearity, and the relative error in with a thermocouple was $<1 \%$ [14]. The cavity lengths drift of $1 \mu \mathrm{m}$ to $4 \mu \mathrm{m}$ for an FP sensor was observed by Cheymol et al. [15] after a total dose of 4.5 GGy gamma radiation. A few researchers have reported on all-fiber FP sensors in the radiation environments, and there have been no investigations into pressure sensing characteristics.

In this paper, the sensing characteristics of the FBG and all-fiber FP sensors were investigated following high levels of radiation up to $2 \mathrm{MGy}$. It was found that the RI-BWS of the B-FBG was -62 $\mathrm{pm}$ under radiation, and the temperature sensitivity of FBGs was unstable after irradiation. The pressure sensitivity deviation of the A-FP before and after irradiation was small and the pressure sensitivity was stable, although the wavelength shift caused by irradiation was $243.1 \mathrm{pm}$.

\section{Sample preparation}

To compare the radiation resistance characteristics of sensors made of different fibers, three single-mode fibers (SMFs) were used in our experiment, hereafter referred to as Fiber A, Fiber B, and Fiber $C$. Details of these fibers are shown in Table 1. Although Fibers A and B were radiation-resistant fibers produced by different manufacturers, both were pure silica core with fluorine-doped claddings. Fiber $\mathrm{C}$ was a standard single-mode fiber with a core of germanium-doped, pure silica cladding. Except for its coating, Fiber B was polyimide, while the others were acrylate.

The FBGs were written point-by-point in fibers using infrared femtosecond laser, as FBGs written by femtosecond laser have better stability. The length, reflectivity, and full width at half maxima (FWHM) of the FBG were $3 \mathrm{~mm}, 50 \%$, and $0.4 \mathrm{~nm}$, respectively. For the temperature change of $\Delta T$, the responding wavelength shift is $\Delta \lambda_{\mathrm{BT}}=\lambda_{B}(1+\xi) \Delta T$, where $\xi$ is the fiber thermo-optic coefficient [16]. It means that the temperature sensitivity of the FBG is determined by the fiber material. For the silica fiber, the wavelength-temperature sensitivity of $1550 \mathrm{~nm}$ is $13 \mathrm{pm} /{ }^{\circ} \mathrm{C}$. The FBG samples written in Fiber A, Fiber $\mathrm{B}$, and Fiber $\mathrm{C}$ will henceforth be referred to as A-FBG, B-FBG, and C-FBG, respectively. Figures 1(a) and 1(c) are the micrograph and reflection spectra of A-FBG, respectively.

The FP cavities were fabricated using a $157 \mathrm{~nm}$ laser micromachining system. First, using a circle mask, a microhole was drilled in the fiber endface. Next, a ring-shaped mask was used to machine a gutter structure. Then, the FP cavity was spliced with SMF to form the FP interferometer. The FP interferometer was mainly an air cavity structure. The FP sensors were sensitive to pressure and insensitive to temperature, and the related sensing principle of which has been described in [17]. The FP sample was only fabricated in Fiber A and called A-FP. Figures 1(b) and 1(d) are micrograph and reflection spectra of the all-fiber FP interferometer, respectively.

The samples, three FBGs, written in the three types of fiber, and one FP, were encapsulated for the irradiation experiment. The RI-BWS of the FBG sensors was reduced by shielding them with stainless steel tubing [18], so that the FBGs could be inserted into stainless steel tubes with an inner diameter of $1 \mathrm{~mm}$ and outer diameter of $3 \mathrm{~mm}$. Furthermore, the FP was inserted into a stainless steel component with thread for the pressure experiment. Experimental samples were not subjected to thermal treatment or $\mathrm{H}_{2}$-loading pretreatment before the irradiation experiment. 


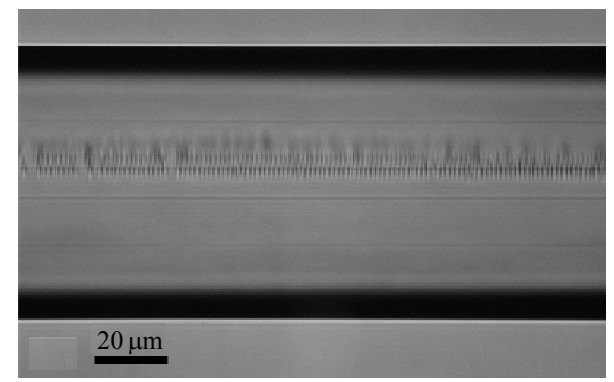

(a)

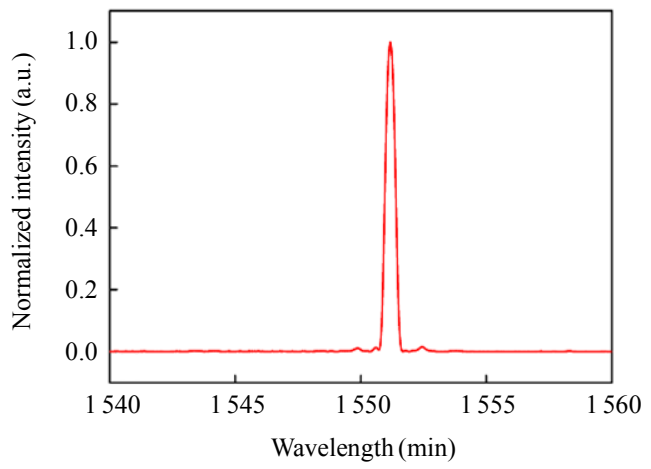

(c)

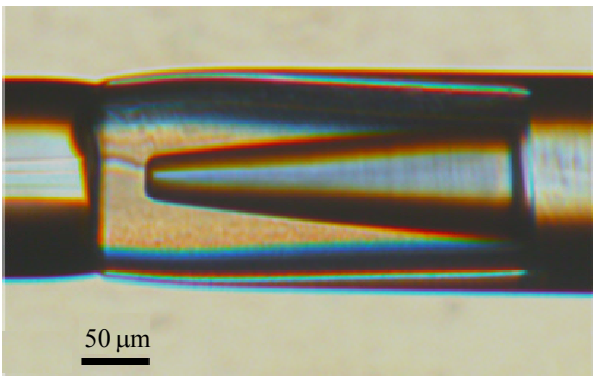

(b)

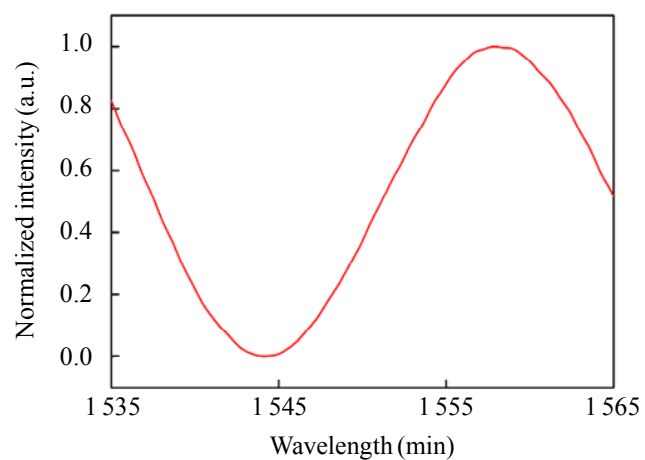

(d)

Fig. 1 Micrographs of (a) FBG and (b) FP, and reflection spectra of (c) FBG and (d) FP.

Table 1 Main specifications of fibers.

\begin{tabular}{ccccc}
\hline Name & Fiber type & Core & Cladding & Coating \\
\hline Fiber A & SMF & Pure silica & $\begin{array}{c}\text { Fluorine-doped } \\
\text { silica }\end{array}$ & Acrylate \\
Fiber B & SMF & Pure silica & $\begin{array}{c}\text { Fluorine-doped } \\
\text { silica }\end{array}$ & Polyimide \\
Fiber C & SMF & $\begin{array}{c}\text { Germanium-doped } \\
\text { silica }\end{array}$ & Pure silica & Acrylate \\
\hline
\end{tabular}

\section{Experimental procedure}

During irradiation, the wavelength shift of the B-FBG was monitored in situ. The experimental setup is shown in Fig. 2, and the wavelength shift and reflection spectra were recorded by an optical spectrum analyzer (OSA) and a computer placed $45 \mathrm{~m}$ away from the gamma radiation source. The $40 \mathrm{~m}$ fiber was located outside the irradiated area, with only $5 \mathrm{~m}$ located in the irradiated area. Since the fiber in the irradiated area was too short, the transmission loss of the fiber induced by irradiation could be ignored. The samples were fixed on a stainless steel plate placed horizontally in the radiation facility to ensure that they were exposed to the same dose rate.

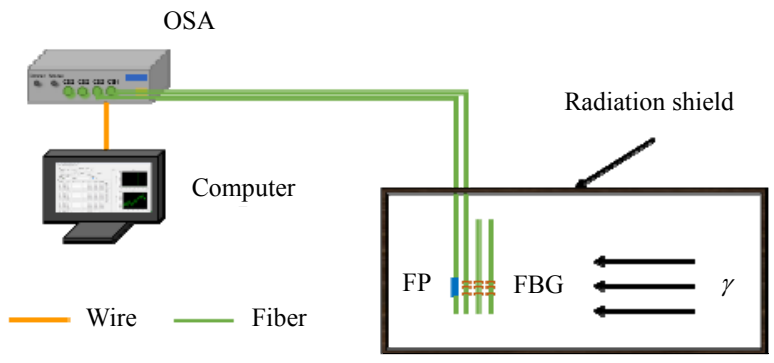

Fig. 2 Irradiation experimental set-up.

The temperature of the radiation environment was $(44 \pm 1){ }^{\circ} \mathrm{C}$ throughout the procedure, continuously monitored by a thermocouple affixed closely to the samples. The temperature outside the radiation environment was room temperature. In the radiation environment, the dose rate for the samples was set to $6499.4 \mathrm{~Gy} / \mathrm{h}$, the total time was set to $330 \mathrm{~h}$ and $56 \mathrm{~min}$, and the accumulated dose was set to exceed $2 \mathrm{MGy}$.

The reflective spectra of the B-FBG are shown in Fig. 3(a) before irradiation, at a $1 \mathrm{MGy}$ dose and a 2 MGy dose. The initial Bragg wavelength of the FBG was approximately $1551.172 \mathrm{~nm}$. The 
normalized peak intensity of the reflection decreased as the radiation dose increased. It was possible that defects in the fiber form color centers were due to gamma radiation, and the RIA of fiber increased [19, 20]. Moreover, radiation had no effect on FWHM, which was $0.4 \mathrm{~nm}$ before irradiation, at a $1 \mathrm{MGy}$ dose and a 2 MGy dose.

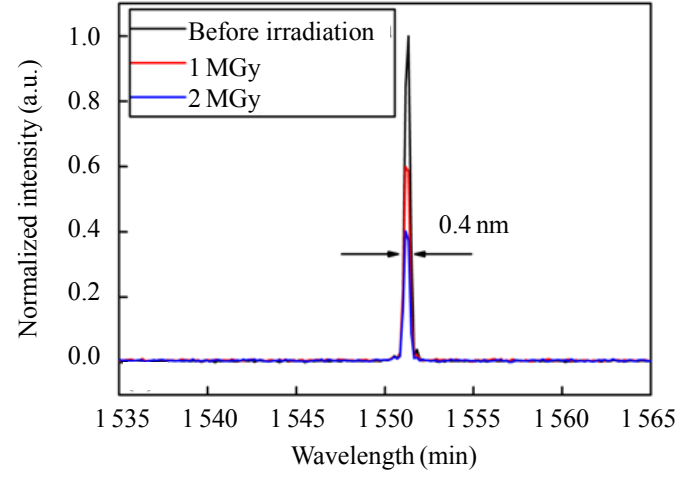

(a)

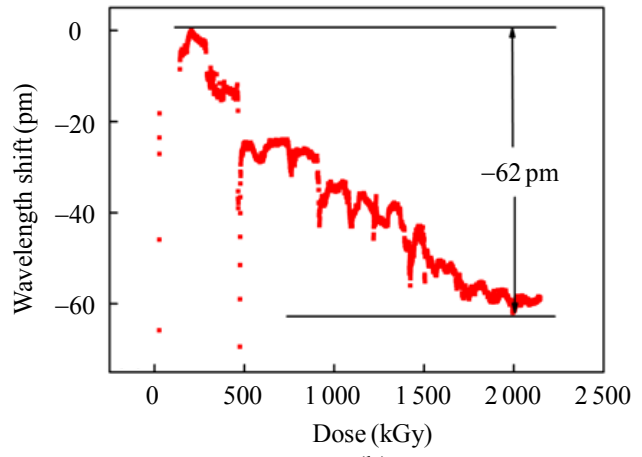

(b)

Fig. 3 Spectra and RI-BWS of B-FBG during irradiation: (a) spectra and (b) RI-BWS.

The RI-BWS of B-FBG was monitored during irradiation in Fig. 3(b). Since the temperature of the radiation environment was above room temperature, the Bragg wavelength of B-FBG had a redshift at the beginning of irradiation. Similarly, its Bragg wavelength at a $473.4 \mathrm{kGy}$ dose in Fig. 3(b) had a blueshift as it was removed from the radiation environment for adjustment, and had a redshift then returned rapidly. In the irradiation experiment, the data for $30 \mathrm{kGy}-140 \mathrm{kGy}$ was missing, as the fiber was broken. The RI-BWS of the B-FBG was $-62 \mathrm{pm}$ at the same temperature and tended to saturate under $2 \mathrm{MGy}$ gamma radiation. The phenomenon resulted from the change in the refractive index modulation and the period of the FBG, which was induced mainly by the change in the refractive index and the density of the fiber under gamma radiation, respectively [21-24].

The spectra of A-FP are shown in Fig. 4 before irradiation, at a $1 \mathrm{MGy}$ dose and a $2 \mathrm{MGy}$ dose. This graph indicates that the free spectral range of FP decreased with increasing radiation dose. Thus, as the radiation dose increased, the cavity length of the FP increased. The cavity length of FP was $48.973 \mu \mathrm{m}$ and $48.981 \mu \mathrm{m}$ before and after $2 \mathrm{MGy}$ gamma irradiation, respectively. This means that radiation increased the FP cavity length by $8 \mathrm{~nm}$. This may have been caused by the compaction of the fiber due to change in fiber density [24].

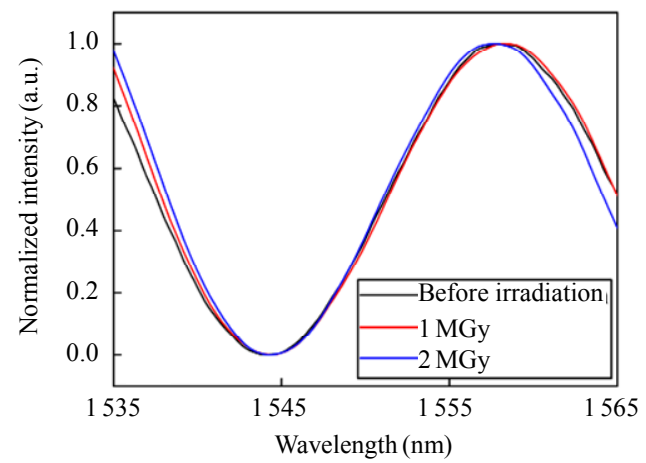

Fig. 4 Spectra of A-FP during irradiation.

\section{Results and discussion}

\subsection{FBG sensors}

For FBG samples, the Bragg wavelengths were recorded at $50^{\circ} \mathrm{C}$ in the laboratory before and after irradiation. Table 2 shows the shift in FBG caused by irradiation. B-FBG was broken after irradiation. The shifts of A-FBG and C-FBG were $+26.2 \mathrm{pm}$ and $+223.6 \mathrm{pm}$, respectively. The redshift of A-FBG after irradiation was small, due to the pure silica core, and the fluorine-doped cladding of Fiber A. Fiber A formed fewer color centers than Fiber C during irradiation [19], consequently increasing the radiation resistance. The refractive index modulation and the period of the FBG changed with increasing radiation dose, thus the Bragg wavelengths changed at the same temperature. 
Table 2 Shift of irradiated FBG samples.

\begin{tabular}{cccc}
\hline \multirow{2}{*}{ Samples } & \multicolumn{2}{c}{$\lambda_{B}(\mathrm{~nm})$} & \multirow{2}{*}{ Shift $(\mathrm{pm})$} \\
& Before irradiation & After irradiation & \\
\hline A-FBG & 1550.3118 & 1550.3380 & +26.2 \\
C-FBG & 1550.3327 & 1550.5563 & +223.6 \\
\hline
\end{tabular}

After irradiation, temperature experiments were performed on both irradiated and non-irradiated FBG samples, and the results of the first temperature experiment are shown in Table 3. The temperature sensitivity deviations of A-FBG and C-FBG between irradiated and non-irradiated were $0.0789 \%$ and $0.5734 \%$, respectively. The differences in the temperature sensitivity between the irradiated and non-irradiated FBGs may have been induced by the changes in the thermo-optic coefficient of the fiber, which resulted from the variation in fiber residual stress induced by irradiation [25]. Moreover, the temperature sensitivity deviation of A-FBG was smaller than that of C-FBG, as Fiber A had better radiation resistance than Fiber $\mathrm{C}$ owing to the undoped-germanium fiber forming fewer color centers.

Table 3 Comparison of temperature characteristics between non-irradiated and irradiated FBG samples.

\begin{tabular}{cccc}
\hline & Samples & $\begin{array}{c}\text { Temperature sensitivity } \\
\left(\mathrm{pm} /{ }^{\circ} \mathrm{C}\right)\end{array}$ & $\begin{array}{c}\text { Sensitivity } \\
\text { deviation (\%) }\end{array}$ \\
\hline \multirow{2}{*}{ A-FBG } & Not irradiation & 12.673 & 0.0789 \\
& Irradiation & 12.663 & \\
C-FBG & Not irradiation & 12.730 & 0.5734 \\
\hline
\end{tabular}

Irradiated and non-irradiated samples were subjected to repeated temperature experiments to obtain the influence of radiation on the sensing stability of the sensors. Temperature characteristics of these FBGs were investigated from $50{ }^{\circ} \mathrm{C}$ to $350{ }^{\circ} \mathrm{C}$ with intervals of $30{ }^{\circ} \mathrm{C}$. The results of the A-FBG are shown as an example in Fig. 5. It can be seen that three measurement curves of the non-irradiated sample coincided. However, the temperature sensitivity curves of irradiated sample did not coincide during temperature experiments. Only the temperature sensitivity of the first experiment was consistent with that of the non-irradiated sample. The temperature sensitivity of the non-irradiated sample was stable, but that of the irradiated sample was unstable. It was likely that radiation caused an unrecoverable variation in the refractive index modulation of FBG. Moreover, Figure 5(b) shows that the fourth and fifth temperature response curve trends were consistent. The reason may be that the change of residual stress caused by irradiation was partially recovered after multiple thermal annealing. Hence, the next step in this research will be to improve sensing stability and reduce RI-BWS. If there is a processing method that makes the temperature sensitivity stable and the RI-BWS accurately calibrated, the FBG sensors have great potential applications in the radiation environment.

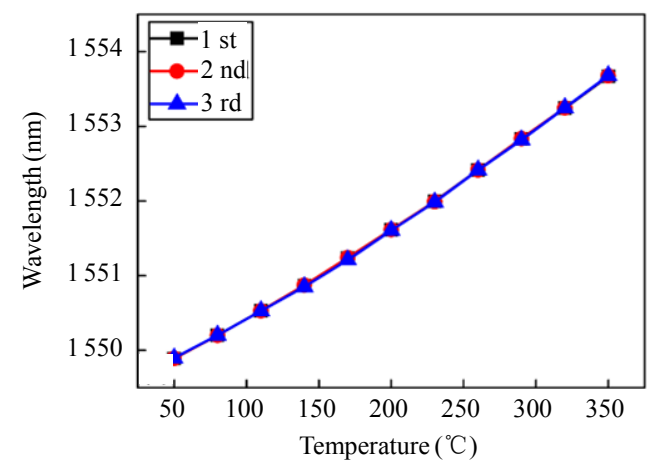

(a)

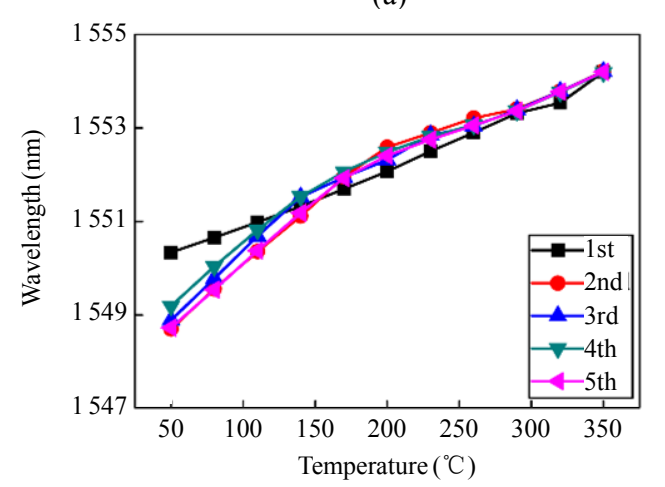

(b)

Fig. 5 Temperature characteristic of (a) the non-irradiated sample and (b) the irradiated sample.

\subsection{FP sensors}

Pressure experiments were performed on the FP sample before and after irradiation to compare sensing characteristics. The comparison of the pressure sensing characteristics of A-FP at room 
temperature before and after irradiation is presented in Table 4. It shows the A-FP shifted $+243.1 \mathrm{pm}$ before and after irradiation, resulting from the variation in its cavity length, which was induced by the density change of the fiber material during irradiation [24]. The pressure sensitivity deviation of the A-FP before and after irradiation was remarkably small, only $0.0834 \%$, as the radiation had little impact on the air cavity of the FP.

Table 4 Comparison of pressure characteristics of the FP sample before and after irradiation.

\begin{tabular}{ccccc}
\hline Sample & $\begin{array}{c}\text { Pressure sensitivity } \\
(\mathrm{pm} / \mathrm{MPa})\end{array}$ & $\begin{array}{c}\text { Sensitivity } \\
\text { deviation }(\%)\end{array}$ & $\begin{array}{c}\text { Shift } \\
(\mathrm{pm})\end{array}$ \\
\hline \multirow{2}{*}{ A-FP } & Before irradiation & -176.067 & 0.0834 & +243.1 \\
& After irradiation & -176.214 & 0 \\
\hline
\end{tabular}

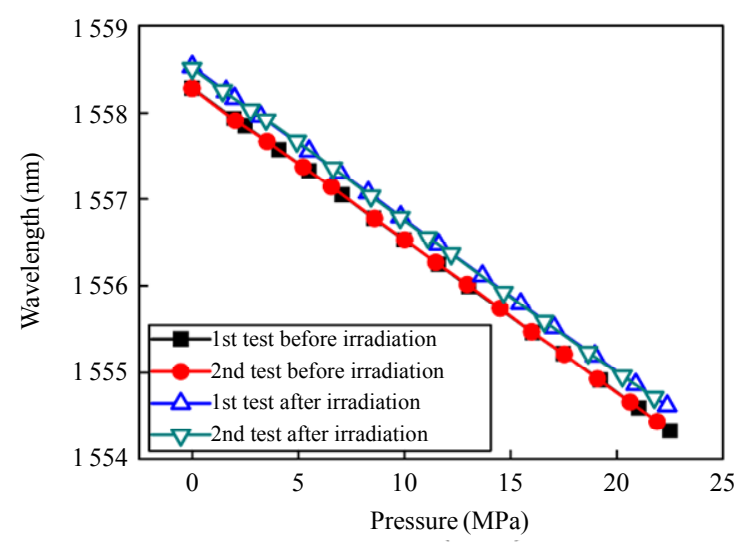

Fig. 6 Pressure characteristic of A-FP.

The FP sample was subjected to two pressure experiments before and after irradiation. The results for the pressure characteristic of A-FP are shown in Fig. 6. The pressure sensitivity curves of the irradiated sample coincided and the pressure sensitivity coefficient was stable. Since radiation may affect some properties of the materials, the air structure of the FP cavity reduced this effect and improved radiation resistance. Nevertheless, the wavelength of the FP showed a shift after irradiation. If the wavelength shift can be accurately calibrated, then the FP pressure sensors have a potential application in the radiation environment.

\section{Conclusions}

In summary, we investigated the radiation response of FBGs, written point-by-point with an infrared femtosecond laser in three types of SMF, and FPs fabricated by $157 \mathrm{~nm}$ laser. Although the sensors performed well in the non-irradiated environment, the RI-BWS of the B-FBG was $-62 \mathrm{pm}$ under a $2 \mathrm{MGy}$-dose gamma radiation. Peak intensity of the B-FBG decreased as the radiation dose increased, although radiation had no effect on FWHM. The redshift of the A-FBG was $26.2 \mathrm{pm}$ at $50{ }^{\circ} \mathrm{C}$ before and after irradiation. The temperature sensitivity deviation of A-FBG between irradiated and non-irradiated conditions was $0.0789 \%$. This deviation was small, but the temperature sensitivity curves of the irradiated sample did not coincide and the temperature sensitivity was unstable. Radiation had little effect on the pressure sensitivity of the FP sensors. The pressure sensitivity deviation of the A-FP was $0.0834 \%$ before and after irradiation. The pressure sensitivity curves of the irradiated sample coincided and the pressure sensitivity coefficient was stable. However, the wavelength of the FP sample displayed a shift before and after irradiation.

The FBG sensors fabricated on different fibers had different radiation responses. Fiber materials, purities, and manufacturing processes may have the affected radiation resistance of the fiber sensors. For FP, the FP sensor consisted of an air cavity, which made imparted a greater radiation resistance, and therefore, offered the possibility of the application of the FP pressure sensors in the gamma radiation environment. FBG sensors need further research into their suitability for nuclear radiation environmental applications. The next step in this research should be to study the radiation effect of the FBG with different parameters, such as gratings length, reflectivity, and FWHM, and explore new methods of improving sensing stability, reducing RI-BWS and further improving the radiation resistance of the FP cavities.

\section{Acknowledgment}

This work was funded by the National Science 
Foundation of China (NCSF) (Grant No. 51875091), the Study and Application of Full-model Impact Dynamic Fretting Damage Test System in the Extreme Environment (Grant No. 51627806), Research on Application of Optical Fiber Sensing in Nuclear Power (Grant No. 180046), Optical Fiber Sensing and Processing Prototype for Nuclear Field Key Parameter Measurement (Grant No. 191091), Data Acquisition and Post-processing Software Development for Integrated Fiber Optic Sensors (Grant No. 190167), and the State 111 Project (Grant No. B14039).

Open Access This article is distributed under the terms of the Creative Commons Attribution 4.0 International License (http://creativecommons.org/licenses/by/4.0/), which permits unrestricted use, distribution, and reproduction in any medium, provided you give appropriate credit to the original author(s) and the source, provide a link to the Creative Commons license, and indicate if changes were made.

\section{References}

[1] C. Petrie, B. Wilson, and T. Blue, "In situ gamma radiation-induced attenuation in sapphire optical fibers heated to $1000^{\circ} \mathrm{C}$," Journal American Ceramic Society, 2014, 97(10): 3150-3156.

[2] C. Cangialosi, S. Girard, M. Cannas, A. Boukenter, E. Marin, S. Agnello, et al., "On-line characterization of gamma radiation effects on single-ended Raman based distributed fiber optic sensor," IEEE Transactions on Nuclear Science, 2016, 63(4): 2051-2057.

[3] X. Pheron, J. Bertrand, S. Girard, Y. Ouerdane, S. Delepine-Lesoille, and A. Boukenter, "Brillouin scattering based sensor in high gamma dose environment: design and optimization of optical fiber for long-term distributed measurement," SPIE, 2012, 8421: 8421A4.

[4] A. Gusarov, F. Berghmans, O. Deparis, A. Fernandez, Y. Defosse, P. Megret, et al., "High total dose radiation effects on temperature sensing fiber Bragg gratings," IEEE Photonics Technology Letters, 1999, 11(9): 1159-1161.

[5] K. Krebber, H. Henschel, and U. Weinand, "Fibre Bragg gratings as high dose radiation sensors?" Measurement Science and Technology, 2006, 17(5): 1095-1102.

[6] M. Zaghloul, M. Wang, S. Huang, C. Hnatovsky, D. Grobnic, S. Mihailov, et al., "Radiation resistant fiber Bragg grating in random air-line fibers for sensing applications in nuclear reactor cores," Optics Express, 2018, 26(9): 11775-11786.

[7] L. Remy, G. Cheymol, A. Gusarov, A. Morana, E. Marin, and S. Girard, "Compaction in optical fibres and fibre Bragg gratings under nuclear reactor high neutron and gamma fluence," IEEE Transactions on Nuclear Science, 2016, 63(4): 2317-2322.

[8] Y. Xu, L. Ma, S. Jiang, and Z. He, "Effect of kGy dose level gamma radiation on Ge-doped FBGs and femtosecond-laser-inscribed pure-silica-core FBGs," in International Conference on Optical Communications and Networks, China, Aug. 7-10, 2017.

[9] A. Gusarov, D. Starodubov, F. Berghmans, O. Deparis, Y. Defosse, A. Fernandez, et al., "Comparative study of MGy dose level $\gamma$-radiation effect on FBGs written in different fibres," SPIE, 1999, 3746: 37460A.

[10] H. Henschel, S. Hoeffgen, K. Krebber, J. Kuhnhenn, and U. Weinand, "Influence of fiber composition and grating fabrication on the radiation sensitivity of fiber Bragg gratings," IEEE Transactions on Nuclear Science, 2008, 55(4): 2235-2242.

[11] H. Henschel, S. K. Hoeffgen, J. Kuhnhenn, and U. Weinand, "Influence of manufacturing parameters and temperature on the radiation sensitivity of fiber Bragg gratings," IEEE Transactions on Nuclear Science, 2010, 57(4): 2029-2034.

[12] M. Perry, P. Niewczas, and M. Johnston, "Effects of neutron-gamma radiation on fiber Bragg grating sensors: a review," IEEE Sensors Journal, 2012, 12(11): 3248-3257.

[13] H. Liu, D. Miller, and J. Talnagi, "Fabry-Perot fiber optic sensors in harsh environments," SPIE, 2002, 4772: 118-128.

[14] H. Liu, D. Miller, and J. Talnagi, “Gamma radiation resistant Fabry-Perot fiber optic sensors," Review of Science Instruments, 2002, 73(8): 3112-3118.

[15] G. Cheymol, A. Gusarov, S. Gaillot, C. Destouches, and N. Caron, "Dimensional measurements under high radiation with optical fibre sensors based on white light interferometry-report on irradiation tests," IEEE Transactions on Nuclear Science, 2014, 61(4): 2075-2081.

[16] Y. Rao, "In-fibre Bragg grating sensors," Measurement Science and Technology, 1997, 8(4): 355-375.

[17] T. Yang, Z. Ran, X. He, Z. Li, Z. Xie, B. Wang, et al., "Temperature-compensated multifunctional all-fiber sensors for precise strain/high-pressure measurement," Journal of Lightwave Technology, 2019, 37(18): 4634-4642.

[18] A. Pal, A. Dhar, A. Ghosh, R. Sen, B. Hooda, V. Rastogi, et al., "Sensors for harsh environment: radiation resistant FBG sensor system," Journal of Lightwave Technology, 2017, 35(16): 3393-3398.

[19] S. Girard, J. Keurinck, A. Boukenter, J. P. Meunier, Y. 
Ouerdane, B. Azais, et al., "Gamma-rays and pulsed $\mathrm{X}$-ray radiation responses of nitrogen-, germanium-doped and pure silica core optical fibers," Nuclear Instruments \& Methods in Physics Research B-Beam, 2004, 215(1-2): 187-195.

[20] A. Tomashuk, M. Salgansky, P. Kashaykin, V. Khopin, A. Sultangulova, K. Nishchev, et al., "Enhanced radiation resistance of silica optical fibers fabricated in high $\mathrm{O}_{2}$ excess conditions," Journal of Lightwave Technology, 2014, 32(2): 213-219.

[21] A. Fernandez, B. Brichard, and F. Berghmans, "In situ measurement of refractive index changes induced by gamma radiation in germanosilicate fibers," IEEE Photonics Technology Letters, 2003, 15(10): 1428-1430.

[22] B. Brichard, O. Butov, K. Golant, and A. Fernandez,
"Gamma radiation-induced refractive index change in Ge- and N-doped silica," Journal of Applied Physics, 2008, 103(5): 054905.

[23] S. Kher, S. Chaubey, S. Oak, and A. Gusarov, "Measurement of gamma-Radiation induced refractive index changes in B/Ge doped fiber using LPGs," IEEE Photonics Technology Letters, 2013, 25(21): 2070-2073.

[24] J. Wen, G. Peng, W. Luo, Z. Xiao, Z. Chen, and T. Wang, "Gamma irradiation effect on Rayleigh scattering in low water peak single-mode optical fibers," Optics Express, 2011, 19(23): 23271-23278.

[25] S. Ju, Y. Kim, K. Linganna, Y. H. Kim, and W. Han, "Effect of temperature and gamma-ray irradiation on optical characteristics of fiber Bragg grating inscribed radiation-resistant optical fiber," Photonic Sensors, 2020, 10(1): 16-33. 